\section{OPEN ACCESS}

Edited by:

Freimut Dankwart Juengling,

St. Claraspital Basel, Switzerland

Reviewed by: Gerard R. Ridgway,

Perspectum Diagnostics,

United Kingdom

Alessandro Tessitore,

Università degli Studi della Campania

"Luigi Vanvitelli" Caserta, Italy

*Correspondence:

Sirio Cocozza

sirio.cocozza@unina.it

tThese authors have contributed equally to this work.

Specialty section:

This article was submitted to

Applied Neuroimaging,

a section of the journal

Frontiers in Neurology

Received: 17 April 2018

Accepted: 26 July 2018

Published: 17 August 2018

Citation:

Pontillo G, Cocozza S, Brunetti A, Brescia Morra V, Riccio E, Russo C,

Saccà F, Tedeschi E, Pisani A and

Quarantelli M (2018) Reduced

Intracranial Volume in Fabry Disease:

Evidence of Abnormal

Neurodevelopment?

Front. Neurol. 9:672.

doi: 10.3389/fneur.2018.00672

\title{
Reduced Intracranial Volume in Fabry Disease: Evidence of Abnormal Neurodevelopment?
}

\begin{abstract}
Giuseppe Pontillo ${ }^{1 \dagger}$, Sirio Cocozza ${ }^{1 * t}$, Arturo Brunetti ${ }^{1}$, Vincenzo Brescia Morra ${ }^{2}$, Eleonora Riccio ${ }^{3}$, Camilla Russo ${ }^{1}$, Francesco Saccà ${ }^{2}$, Enrico Tedeschi ${ }^{1}$, Antonio Pisani ${ }^{3}$ and Mario Quarantelli ${ }^{4}$ on behalf of the AFFINITY study group
\end{abstract}

\begin{abstract}
${ }^{1}$ Department of Advanced Biomedical Sciences, University "Federico II," Naples, Italy, ${ }^{2}$ Department of Neurosciences and Reproductive and Odontostomatological Sciences, University "Federico II," Naples, Italy, ${ }^{3}$ Nephrology Unit, Department of Public Health, University "Federico II," Naples, Italy, ${ }^{4}$ Institute of Biostructure and Bioimaging, National Research Council, Naples, Italy
\end{abstract}

Introduction: Lysosomal storage disorders (LSD) are often characterized by abnormal brain development, reflected by a reduction of intracranial volume (ICV). The aim of our study was to perform a volumetric analysis of intracranial tissues in Fabry Disease (FD), investigating possible reductions of ICV as a potential expression of abnormal brain development in this condition.

Materials and Methods: Forty-two FD patients (15 males, mean age $43.3 \pm 13.0$ years) were enrolled along with 38 healthy controls $(\mathrm{HC})$ of comparable age and sex. Volumetric MRI data were segmented using SPM12 to obtain intracranial tissue volumes, from which ICV values were derived.

Results: Mean ICV of FD patients was $8.1 \%$ smaller compared to the control group $\left(p<5 \cdot 10^{-5}\right)$. Unlike what typically happens in neurodegenerative disorders, no significant differences emerged when comparing between the two groups the fractional volumes of gray matter, white matter and CSF (i.e., normalized by ICV), consistent with a harmonious volumetric reduction of intracranial structures.

Discussion: The present results suggest that in FD patients an abnormality of brain development is present, expanding the current knowledge about central nervous system involvement in FD, further emphasizing the importance of an early diagnosis.

Keywords: Fabry disease, neurodevelopmental disorders, magnetic resonance imaging, brain, atrophy

\section{INTRODUCTION}

Fabry disease (FD) is a rare, $\mathrm{X}$-linked disorder characterized by a progressive accumulation of globotriaosylceramide (Gb3) and related glycosphingolipids in different cells $(1,2)$. In contrast to many other lysosomal storage disorders (LSD) $(3,4)$, FD is regarded as a clinical adult-onset condition, with most patients remaining asymptomatic in the first years of life and then manifesting the characteristic multisystemic signs of the disease (2).

The use of advanced MRI techniques has recently suggested that different mechanisms of brain damage could be present in FD, with the vision of this disorder slowly changing from a purely cerebrovascular disease to a condition characterized by a more global and multifaceted cerebral involvement (5-9). 
Of note, the physiopathology of CNS alterations in FD has not yet been completely understood. Along with vascular and neurodegenerative mechanisms, both due to glycosphingolipid accumulation, the possibility that in FD, similarly to other LSD, an abnormal development of CNS could occur has never been fully investigated.

Indeed, although several studies were conducted to investigate possible changes in relative brain tissue (especially gray matter GM-) volumes in $\mathrm{FD}(7,8,10)$, to the best of our knowledge, no study has focused on the evaluation of total intracranial volume (ICV). This latter measure represents a proxy for the maximal brain growth obtained during development and maturation $(11,12)$, whose changes may represent a possible indicator of neurodevelopmental anomaly.

With this knowledge, aim of our study was to perform a volumetric analysis of intracranial tissues in $\mathrm{FD}$, investigating possible differences in terms of ICV between FD patients and a group of healthy controls (HC) as a possible expression of abnormal brain development in this condition.

\section{MATERIALS AND METHODS}

\section{Participants}

Brain MRI studies of 42 patients with genetically proven FD, no history of any cerebrovascular events and classical phenotype of the disease were analyzed, along with those from $38 \mathrm{HC}$ of comparable age and sex. For both groups exclusion criteria included a history of neurologic or major psychiatric disorders and systemic diseases that may affect the CNS (e.g., uncontrolled endocrine disorder), as well as the presence of any significant cerebral focal lesion on brain MRI.

Demographic and clinical information of all the subjects included in the analysis are listed in Table 1, while genetic findings are reported in Table s1 of the Supplementary Materials.

\section{Standard Protocol Approvals, Registrations, and Patient Consents}

All participants gave informed written consent for use of their images for research purposes. The study was approved by the local institutional review board "Carlo Romano," in accordance to the Declaration of Helsinki.

\section{MRI Data Acquisition}

All MRI exams have been carried out on the same 3 Tesla MR scanner (Trio, Siemens Medical Systems, Erlangen, Germany). The sequences used for this study included a FLAIR sequence for assessment of White Matter Hyperintensities (WMH) and an isotropic T1w acquisition for the volumetric analysis, acquired during the same scan session. Details of the acquired sequences are reported in the Supplementary Materials.

Abbreviations: LSD, Lysosomal Storage Disorder; ICV, Intracranial Volume; FD, Fabry Disease; HC, Healthy Control; WMH, White Matter Hyperintensities; fGM, fractional Gray Matter; fWM, fractional White Matter; fCSF, fractional CerebroSpinal Fluid; ERT, Enzyme-Replacement Therapy.
TABLE 1 | Participants' demographic and clinical variables.

\begin{tabular}{llc}
\hline & HC & FD \\
\hline Age $($ mean $\pm S D)$ & $43.2 \pm 14.5$ & $43.3 \pm 13.0$ \\
Sex $(F-M)$ & $23-15$ & $27-15$ \\
ERT & $n / a$ & $31 / 42$ \\
ERT duration (mean $\pm S D)$ & $n / a$ & $51.8 \pm 53.4$ \\
Hypertension & $n / a$ & $11 / 42$ \\
Arrhythmia & $n / a$ & $2 / 42$ \\
Left ventricular hypertrophy & $n / a$ & $31 / 42$ \\
Renal failure & $n / a$ & $14 / 42$ \\
Proteinuria & $n / a$ & $22 / 42$ \\
Neuropathy & $n / a$ & $30 / 42$ \\
Acroparesthesia & $n / a$ & $29 / 42$ \\
Gastrointestinal involvement & $n / a$ & $12 / 42$ \\
Cornea verticillata & $n / a$ & $33 / 42$ \\
Angiocheratoma & $n / a$ & $36 / 42$ \\
\hline
\end{tabular}

ERT, enzyme replacement therapy; FD, Fabry disease; HC, healthy control; n/a, not applicable. Ages are expressed in years, while ERT duration is expressed in months. Renal failure considered present when the estimated glomerular filtration rate of the patient was $<90 \mathrm{~mL} / \mathrm{min}$, while proteinuria was considered present when the patient scored a value $>150 \mathrm{mg} / 24 \mathrm{~h}$.

\section{MRI Data Analysis}

Brain MR scans were visually assessed for the presence of $\mathrm{WMH}$ by two neuroradiologists in consensus, blind to diagnosis. The load of WMH was rated in all subjects, by grading it separately in periventricular and deep hemispheric white matter (WM), with a score ranging from 0 to 3 for each location. The overall score (hereinafter Fazekas' score) was then calculated as the sum of the two values, as proposed by Fazekas et al. within a recent multicenter MR study on FD (13).

Analysis of structural T1-weighted MRI data was conducted using the Statistical Parametric Mapping (SPM12) software package (http://www.fil.ion.ucl.ac.uk/spm).

For ICV and global GM, WM, and cerebrospinal fluid (CSF) volume measures, structural data were processed using the unified segmentation tool (14); and ICV was then computed with the "tissue volumes" utility, by adding up the segmented GM, WM and CSF volumes (15).

In addition, to investigate possible changes of intracranial tissue volumes independent from ICV, fractional volumes of brain tissues were calculated as their ratio to ICV, thus obtaining fractional GM (fGM), fractional WM (fWM), and fractional CSF (fCSF) $(16,17)$.

Finally, to investigate possible regional GM differences between the two groups, a voxel based morphometry (VBM) analysis was carried out. With this aim, the Diffeomorphic Anatomical Registration Through Exponentiated Lie Algebra (DARTEL) algorithm (18) was employed to create a study-specific template for spatial normalization of the segmented images. The resulting flow fields created by the DARTEL procedure were then used to generate spatially normalized, Jacobian scaled, resliced 
(2.0-mm isotropic voxels) and smoothed (8-mm FWHM isotropic Gaussian kernel) GM maps in the standard MNI space (19).

\section{Statistical Analysis}

Differences between FD and HC groups in terms of age and sex were probed by Student $t$-test and by Chi-squared test, respectively, while differences in terms of Fazekas' score were assessed using a Mann-Whitney test. A general linear model (GLM), including age and sex as confounding covariates, was employed to probe possible differences in terms of ICV and fractional intracranial tissue volumes. Finally, correlations of volumes with Fazekas' score and clinical variables, including cardiac and renal functions, as well as ERT duration, were assessed by partial correlation analysis, correcting for age and sex. These analyses were carried out using Statistical Package for Social Science (SPSS) package (SPSS Inc., version 17.0, Chicago, IL), with a two-tailed significance level of $p<0.05$, Bonferroni-corrected.

For the VBM analysis, differences between the two groups and correlations with the Fazekas' score and clinical variables were also assessed by means of the GLM in SPM12, including in the model age and sex, as well as ICV, to assess local differences independent from head size. In comparing the two groups, both contrasts $(\mathrm{HC}>\mathrm{FD}$ and $\mathrm{HC}<\mathrm{FD}$ ) were probed, and differences were considered significant for $p<0.05$, corrected for the familywise error at cluster level.

\section{RESULTS}

The FD and HC groups were not significantly different for age $(43.3 \pm 13.0$ for FD vs. $43.2 \pm 14.5$ for HC; $p=0.96)$, and sex $(\mathrm{M}-\mathrm{F}=27-15$ vs. $23-15$ for FD and HC, respectively; $p=0.73$ ).

Among the FD patients, 20 out of 42 (47.6\%) showed absence of WMH in any regions, 17 patients had a Fazekas' score of 1, 4 patients $(9.5 \%)$ a score between 2 and 3, and only one patient (2.4\%) a high WMH load, scoring a total Fazekas' score of 6. On the other hand, $65.8 \%$ of controls (25 out of 38 subjects) showed absence of significant WMH in any region (Fazekas' score $=0$ ), 12 (31.6\%) had a Fazekas' score of 1 and only one HC (3.1\%) had a Fazekas' score of 2 ( $p=0.07$ at Mann-Whitney test).

When evaluating possible differences in terms of ICV, FD patients showed significantly smaller volumes compared to $\mathrm{HC}$, with a mean ICV that was $8.1 \%$ lower compared to the control group $(1267.8 \pm 121.5 \mathrm{ml}$ vs. $1379.8 \pm 137.2 \mathrm{ml}$ in FD and HC, respectively; $p<5 \cdot 10^{-5}$; $95 \%$ confidence interval of the difference $=-149.1 /-61.4 \mathrm{ml})$ (Figure 1).

No significant differences emerged between the two groups when comparing the fGM $(50.5 \pm 3.6 \%$ vs. $50.1 \pm 3.9 \%$ in FD and $\mathrm{HC}$, respectively; $p=0.41 ; 95 \%$ confidence interval of the difference $=-0.6 \% / 1.4 \%)$, the fWM $(30.6 \pm 2.0 \%$ vs. $31.4 \pm 2.0 \%$ in FD and $\mathrm{HC}$, respectively; $p=0.10 ; 95 \%$ confidence interval of the difference $=-1.6 \% / 0.1 \%)$ and the fCSF $(18.8 \pm 4.4 \%$ vs. 18.5 $\pm 4.0 \%$ in $\mathrm{FD}$ and $\mathrm{HC}$, respectively; $p=0.86 ; 95 \%$ confidence interval of the difference $=-1.0 \% / 1.7 \%)$. The VBM analysis revealed only a cluster of reduced GM density in FD patients compared to $\mathrm{HC}$ at the level of the thalami, bilaterally, extending

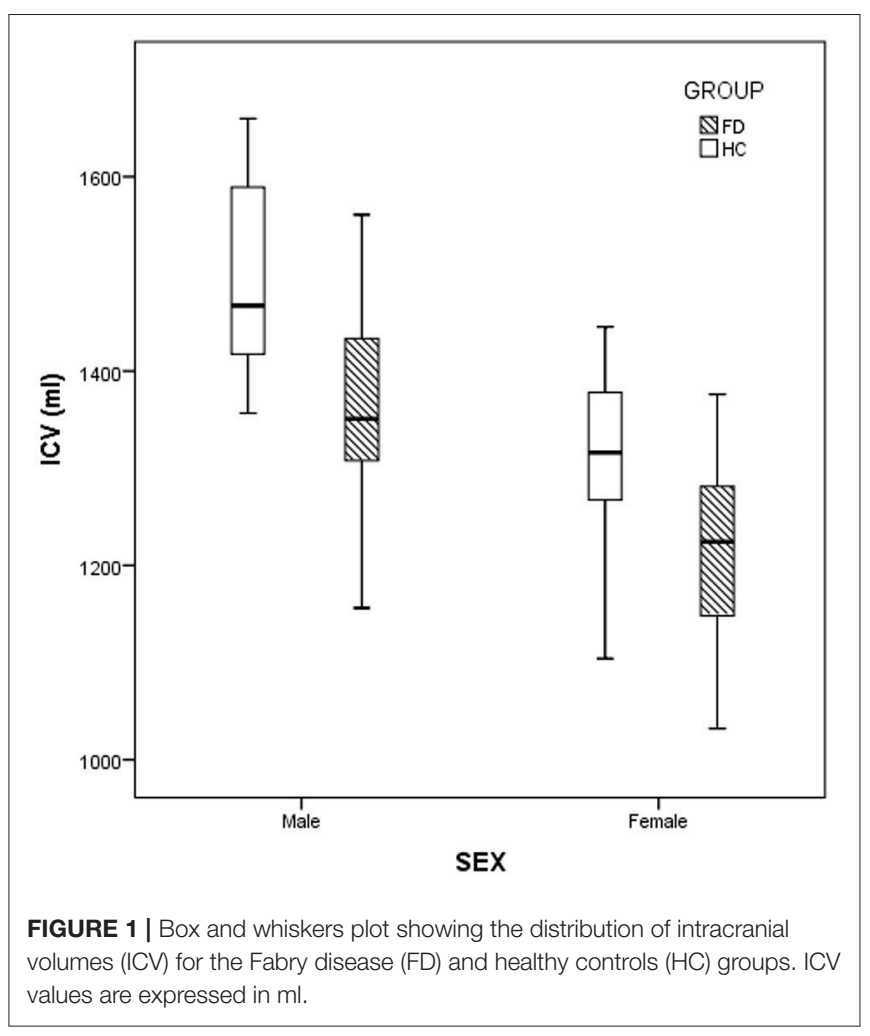

toward the left hippocampus ( $p=0.001)$ (Figure 2; Table s2). No significant clusters of increased GM density were found in FD patients compared to HC.

Finally, when testing the relationship with WMH load and clinical variables, no significant correlation emerged for ICV, fractional intracranial tissue volumes or regional GM density.

\section{DISCUSSION}

We investigated volumetric alterations of intracranial structures in FD patients, demonstrating a smaller ICV in these subjects compared with $\mathrm{HC}$, along with a preservation of fractional intracranial tissue volumes. These results, taken together, demonstrate a harmonious reduction of all intracranial tissue volumes, supporting the hypothesis of a possible abnormality of neural development in these patients.

ICV is defined as the sum of GM, WM, and CSF, and it is a representation of the maximal brain growth obtained during development and maturation $(11,12)$. Indeed, the peak of the ICV is reached early in life, such that, by the age of 6 years, total brain volume is $90 \%$ of adult volume and reaches full adult volume during adolescence $(20,21)$. Once ICV peaks and the skull sutures are completely fused, there is no further change in this measure, regardless of changes that may occur in brain tissues (22), which when occurring must be compensated by changes in the CSF compartment volume.

Most sphingolipidoses, and LSD in general, are characterized by the relatively prominent involvement of CNS, ranging from 


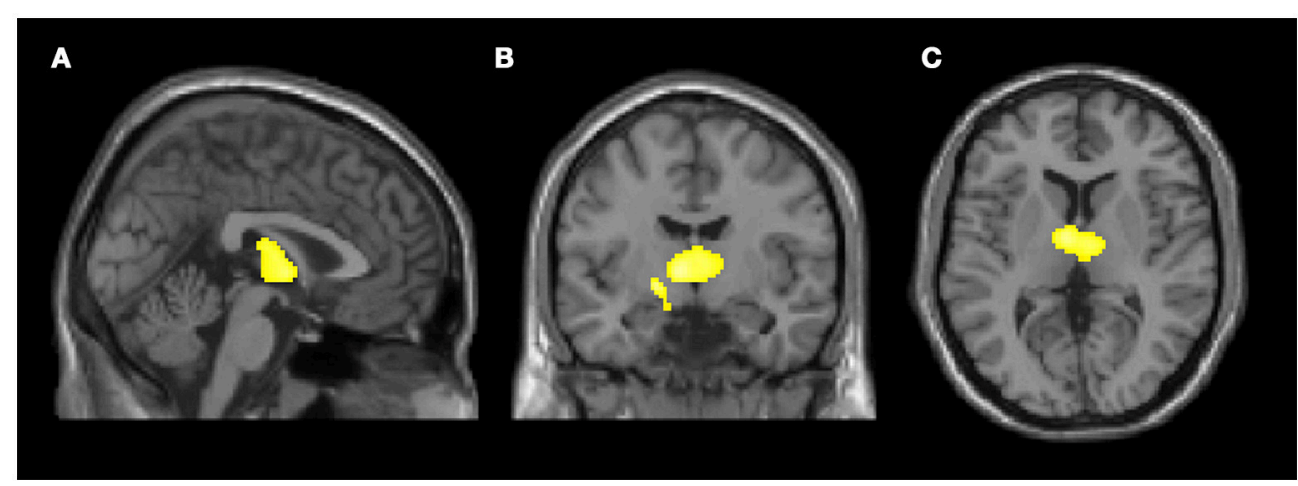

FIGURE 2 | Clusters of gray matter loss in Fabry disease (FD) patients compared to healthy controls (HC). Results are displayed for $p<0.05$ FWE-corrected at cluster level, and superimposed for anatomical reference to multi-planar reconstruction of a single subject T1-weighted volume in the standard Montreal Neurological Institute space (A) sagittal, (B) coronal, (C) axial. No region of increased gray matter volume was present in FD patients compared to HC.

early developmental delay and microcephaly to more subtle neurodevelopmental deficits (23-26).

Interestingly, some studies demonstrated a smaller ICV as an evidence of neurodevelopmental defect not only in early-onset neurodevelopmental disorders but also in other genetic conditions mainly characterized by an adult-onset neurodegeneration, such as Wolfram Syndrome or even prodromal Huntington's Disease $(27,28)$. These findings suggest that in these diseases subtle neurodevelopmental phenomena may occur already in the preclinical phases $(27,28)$.

In our study, we found significantly smaller intracranial volumes in FD patients, a finding that could shed further light on the mechanisms of cerebral involvement in FD, suggesting for the first time that in these patients a neurodevelopmental abnormality may be also present. Indeed, since ICV, once determined by maximal brain growth in childhood, does not change over time, a smaller volume could reflect an incomplete growth of the brain parenchyma, as expression of a global abnormality in the process of brain development (27).

A possible explanation to this finding could be traced to the pathogenic mechanisms typical of FD. Indeed, it may be hypothesized that $\alpha$-galactosidase A could play a role in the normal development of the CNS and, similarly to what happens in many other $\operatorname{LSD}(23,24,26)$, its deficiency could lead to detectable, although mild, neurodevelopmental abnormalities. This speculation, coupled to other evidences demonstrating the possibility of an early detection of clinical and subclinical manifestations in pediatric FD patients $(29,30)$, could further indicate that FD, once considered as an adult-onset condition, is indeed a more complex phenomenon that encompass all ages, also regarding CNS involvement. The present findings thus further stress the importance of a timely diagnosis, or even of newborn screening, and the possible advantages of an early initiation of the enzyme-replacement therapy (ERT) (31). With respect to this speculation, it should also be noted that the absence of a significant correlation with the ERT duration is in line with our hypothesis. Indeed, all FD patients included in the study started the ERT as adults, where this possible neurodevelopmental abnormality could have already developed. To test this hypothesis, however, longitudinal studies including pediatric FD patients should be performed, to further elucidate any possible relationship between ERT and ICV variations.

Along with the evaluation of ICV, we have investigated if different intracranial tissues were affected to a different extent in FD, showing no difference between FD and $\mathrm{HC}$ in terms of fractional intracranial tissue volumes. This finding, coupled to the abovementioned ICV reduction in these patients, indicates the presence of a harmonious volumetric reduction of all intracranial structures in FD patients, rather than a pure neurodegenerative phenomenon. Indeed, neurodegeneration usually affects GM or the entire brain parenchyma volume, determining a reduction of the corresponding fractional volumes, typically with a compensatory increase in CSF volume to maintain total ICV unchanged.

Interestingly, in the post-hoc VBM analysis we found two clusters of reduced GM density involving both thalami with extension to the left hippocampus, in absence of significant correlation between regional GM volume and WMH load. This finding is apparently in conflict with previous volumetric studies, which failed to prove significant regional alterations in GM volumes of FD patients $(8,9)$. A possible explanation to this discrepancy may lay in the segmentation method used in the present work, coupled to the larger sample examined in this work, which provides a slightly greater statistical power, that allowed to detect these subtle differences. Indeed, our analysis was conducted with a different software package (i.e., SPM12) compared to the one used in previous works (e.g., SPM8), which has proved to be one of the most accurate method for intracranial volumes assessment $(15,32)$. Supporting this speculation, an additional analysis performed on the same subjects with SPM8 led to no significant differences at the VBM analysis (data not shown).

Indeed, our VBM findings are also partially in line with previous studies that demonstrated hippocampal atrophy in FD patients as a possible surrogate consequence of a primitive neuronal involvement, independent from brain vasculopathy 
(7, 10). Moreover, similar findings were also reported in other sphingolipidoses such as Niemann-Pick disease, indicating a preferential volume reduction of subcortical gray matter structures in these conditions $(33,34)$. Slightly more prominent thalamic and hippocampal atrophy, in the context of a generalized harmonic reduction of the volumes of all intracranial compartments, could be the expression of an additional abnormal development of subcortical GM or, more probably, of a specific vulnerability to neurodegeneration due to selective neuronal Gb3 accumulation in these structures, which has been reported in anatomopathological studies (7, 35-37). However, longitudinal and neuropsychological studies are needed to further elucidate the physiopathological mechanisms and the clinical meaning of this finding.

It is known that in this condition height is reported to be below the US 50th percentile in young male patients, while no significant difference is described for female patients (29). This evidence could lead to the interpretation of our finding as an expression of a generalized harmonious growth defect, rather than a selective neurodevelopmental abnormality. To test this hypothesis however, we retrospectively retrieved anthropometric measures for the FD group from clinical records, which were not significantly different from the reported values of the adult population of the same geographical area for both males [median height: $170 \mathrm{~cm}$ (range: $158-188 \mathrm{~cm}$ ) vs. $174 \mathrm{~cm}$ in the healthy population; $p=0.18 ; 95 \%$ confidence interval of the difference $=-6.72 / 1.38$ ] and females [median height: $160 \mathrm{~cm}$ (range: $145-175 \mathrm{~cm}$ ); vs. $161 \mathrm{~cm}$ in the healthy population, $p=0.81 ; 95 \%$ confidence interval of the difference $=-2.92 / 2.30$ ] (38). Furthermore, an ancillary partial correlation analysis (corrected for age and sex) proved no significant correlations between height and ICV values $(r=0.225, p=0.169 ; 95 \%$ confidence interval for $r=-0.089 / 0.498)$ in our patients. Although these observations further support our speculation that a reduction of ICV values could be the expression of a neurodevelopmental abnormality, we could not fully test this hypothesis as anthropometric measures were not available in the $\mathrm{HC}$ group, so that further studies are warranted to confirm it.

\section{REFERENCES}

1. Sweeley CC, Klionsky B. Fabry's disease: classification as a sphingolipidosis and partial characterization of a novel glycolipid. J Biol Chem. (1963) 238:3148-50

2. Germain DP. Fabry disease. Orphanet J Rare Dis. (2010) 5:30. doi: 10.1186/1750-1172-5-30

3. Neufeld EF. Lysosomal storage diseases. Ann Rev Biochem. (1991) 60:257-80. doi: 10.1146/annurev.bi.60.070191.001353

4. Staretz-Chacham O, Lang TC, LaMarca ME, Krasnewich D, Sidransky E. Lysosomal storage disorders in the newborn. Pediatrics (2009) 123:1191-207. doi: 10.1542/peds.2008-0635

5. Fellgiebel A, Mazanek M, Whybra C, Beck M, Hartung R, Müller $\mathrm{KM}$, et al. Pattern of microstructural brain tissue alterations in Fabry disease: a diffusion-tensor imaging study. J Neurol. (2006) 253:780-7. doi: 10.1007/s00415-006-0118-y

6. Albrecht J, Dellani PR, Müller MJ, Schermuly I, Beck M, Stoeter P, et al. Voxel based analyses of diffusion tensor imaging in Fabry disease.
In conclusion, we demonstrated that FD patients show a highly significant reduction of the ICV compared to HC, while fractional volumes of intracranial tissues are preserved in this condition. Our results further expand the current knowledge about CNS involvement in FD, suggesting that in these patients an abnormality of brain development could be present, thus emphasizing the importance of an early diagnosis of FD, and possibly of an early ERT initiation.

Further studies are needed to understand if this feature of the disease is part of the spectrum of more generalized growth defect encountered in this pathology, or it represents an independent phenomenon.

\section{AUTHOR CONTRIBUTIONS}

GP and SC: conception, organization, and execution of the research, writing of the manuscript; $\mathrm{AB}$ : organization and execution of the research, writing, and review of the manuscript; VBM: organization and execution of the research, writing of the manuscript; ER: conception and execution of the research, review of the manuscript; CR: conception of the research, writing, and review of the manuscript; FS and ET: organization and execution of the research, review of the manuscript; AP: writing, review, and critique of the manuscript; MQ: design, execution, review, and critique of the statistical analysis, review, and critique of the manuscript.

\section{ACKNOWLEDGMENTS}

Funding by the CNR Strategic Project The Aging: Technological and Molecular Innovations Aiming to Improve the Health of Older Citizens (http://www.progettoinvecchiamento.it) is gratefully acknowledged.

\section{SUPPLEMENTARY MATERIAL}

The Supplementary Material for this article can be found online at: https://www.frontiersin.org/articles/10.3389/fneur. 2018.00672/full\#supplementary-material

J Neurol Neurosurg Psychiatry (2007) 78:964-9. doi: 10.1136/jnnp.2006.1 12987

7. Fellgiebel A, Wolf DO, Kolodny E, Müller MJ. Hippocampal atrophy as a surrogate of neuronal involvement in Fabry disease. $J$ Inherited Metab Dis. (2012) 35:363-7. doi: 10.1007/s10545-0119390-9

8. Paavilainen T, Lepomäki V, Saunavaara J, Borra R, Nuutila P, Kantola I, et al. Diffusion tensor imaging and brain volumetry in Fabry disease patients. Neuroradiology (2013) 55:551-8. doi: 10.1007/s00234-012-1131-8

9. Cocozza S, Pisani A, Olivo G, Saccà F, Ugga L, Riccio E, et al. Alterations of functional connectivity of the motor cortex in Fabry disease: an RS-fMRI study. Neurology (2017) 88:1822-9. doi: 10.1212/WNL.0000000000003913

10. Lelieveld IM, Böttcher A, Hennermann JB, Beck M, Fellgiebel A. Eight-year follow-up of neuropsychiatric symptoms and brain structural changes in Fabry disease. PLoS ONE (2015) 10:e0137603. doi: 10.1371/journal.pone.0137603

11. Matsumae M, Kikinis R, Mórocz IA, Lorenzo AV, Sándor T, Albert MS, et al. Age-related changes in intracranial compartment volumes in normal adults 
assessed by magnetic resonance imaging. J Neurosurg. (1996) 84:982-91. doi: 10.3171/jns.1996.84.6.0982

12. Sgouros S, Goldin JH, Hockley AD, Wake MJ, Natarajan K, et al. Intracranial volume change in childhood. J Neurosurg. (1999) 91:610-6. doi: 10.3171/jns.1999.91.4.0610

13. Fazekas F, Enzinger C, Schmidt R, Grittner U, Giese AK, Hennerici MG, et al. Brain magnetic resonance imaging findings fail to suspect Fabry disease in young patients with an acute cerebrovascular event. Stroke (2015) 46:1548-53. doi: 10.1161/STROKEAHA.114.008548

14. Ashburner J, Friston KJ. Unified segmentation. NeuroImage (2005) 26:839-51. doi: 10.1016/j.neuroimage.2005.02.018

15. Malone IB, Leung KK, Clegg S, Barnes J, Whitwell JL, Ashburner J, et al. Accurate automatic estimation of total intracranial volume: a nuisance variable with less nuisance. NeuroImage (2015) 104:366-72. doi: 10.1016/j.neuroimage.2014.09.034

16. Rudick RA, Fisher E, Lee JC, Simon J, Jacobs L. Use of the brain parenchymal fraction to measure whole brain atrophy in relapsing-remitting MS. Multiple Sclerosis Collaborative Research Group. Neurology (1999) 53:1698-704. doi: 10.1212/WNL.53.8.1698

17. Vågberg $M$, Granåsen $G$, Svenningsson A. Brain parenchymal fraction in healthy adults-a systematic review of the literature. PLoS ONE (2017) 12:e0170018. doi: 10.1371/journal.pone.0170018

18. Ashburner J. A fast diffeomorphic image registration algorithm. NeuroImage (2007) 38:95-113. doi: 10.1016/j.neuroimage.2007.07.007

19. Ashburner J. Computational anatomy with the SPM software. Magn Reson Imaging (2009) 27:1163-74. doi: 10.1016/j.mri.2009.01.006

20. Giedd JN. Structural magnetic resonance imaging of the adolescent brain. Ann N Y Acad Sci. (2004) 1021:77-85. doi: 10.1196/annals.1308.009

21. Courchesne E, Chisum HJ, Townsend J, Cowles A, Covington J, Egaas B, et al. Normal brain development and aging: quantitative analysis at in vivo MR imaging in healthy volunteers. Radiology (2000) 216:672-82. doi: 10.1148/radiology.216.3.r00au37672

22. Morriss-Kay GM, Wilkie AO. Growth of the normal skull vault and its alteration in craniosynostosis: insights from human genetics and experimental studies. J Anato. (2005) 207:637-53. doi: 10.1111/j.1469-7580.2005.00475.x

23. Hoffmann B, Mayatepek E. Neurological manifestations in lysosomal storage disorders - from pathology to first therapeutic possibilities. Neuropediatrics (2005) 36:285-9. doi: 10.1055/s-2005-872810

24. Martin HR, Poe MD, Reinhartsen D, Pretzel RE, Roush J, Rosenberg A, et al. Methods for assessing neurodevelopment in lysosomal storage diseases and related disorders: a multidisciplinary perspective. Acta Paediatr. (2008) 97:69-75. doi: 10.1111/j.1651-2227.2008.00651.x

25. Nixon RA, Yang DS, Lee JH. Neurodegenerative lysosomal disorders: a continuum from development to late age. Autophagy (2008) 4:590-9. doi: 10.4161 /auto.6259

26. Pastores GM, Maegawa GHB. Neuropathic lysosomal storage disorders. Neurol Clin. (2013) 31:1051-71. doi: 10.1016/j.ncl.2013.04.007

27. Nopoulos PC, Aylward EH, Ross CA, Mills JA, Langbehn DR, Johnson HJ, et al. Smaller intracranial volume in prodromal Huntington's disease: evidence for abnormal neurodevelopment. Brain (2011) 134:137-42. doi: 10.1093/brain/awq280

28. Hershey T, Lugar HM, Shimony JS, Rutlin J, Koller JM, Perantie DC, et al. Early brain vulnerability in Wolfram syndrome. PLOS ONE (2012) 7:e40604. doi: 10.1371/journal.pone.0040604

29. Hopkin RJ, Bissler J, Banikazemi M, Clarke L, Eng CM, Germain DP, et al. Characterization of Fabry disease in 352 pediatric patients in the
Fabry Registry. Pediatr Res. (2008) 64:550-5. doi: 10.1203/PDR.0b013e3181 $83 f 132$

30. Laney DA, Peck DS, Atherton AM, Manwaring LP, Christensen KM, Shankar SP, et al. Fabry disease in infancy and early childhood: a systematic literature review. Genet Med. (2015) 17:323-30. doi: 10.1038/gim.2014.120

31. Pisani A, Visciano B, Roux GD, Sabbatini M, Porto C, Parenti G, et al. Enzyme replacement therapy in patients with Fabry disease: state of the art and review of the literature. Mol Genet Metab. (2012) 107:267-75. doi: 10.1016/j.ymgme.2012.08.003

32. Sargolzaei S, Sargolzaei A, Cabrerizo M, Chen G, Goryawala M, Pinzon-Ardila A, et al. Estimating intracranial volume in brain research: an evaluation of methods. Neuroinformatics (2015) 13:427-41. doi: 10.1007/s12021-015-9266-5

33. Walterfang M, Patenaude B, Abel LA, Kluenemann H, Bowman EA, Fahey $\mathrm{MC}$, et al. Subcortical volumetric reductions in adult Niemann-Pick disease type C: a cross-sectional study. Am J Neuroradiol. (2013) 34:1334-40. doi: 10.3174/ajnr.A3356

34. Masingue M, Adanyeguh I, Nadjar Y, Sedel F, Galanaud D, Mochel F, et al. Evolution of structural neuroimaging biomarkers in a series of adult patients with Niemann-Pick type C under treatment. Orphanet J Rare Dis. (2017) 12:22. doi: 10.1186/s13023-017-0579-3

35. deVeber GA, Schwarting GA, Kolodny EH, Kowall NW, et al. Fabry disease: immunocytochemical characterization of neuronal involvement. Ann Neurol. (1992) 31:409-15. doi: 10.1002/ana.410310410

36. Kaye EM, Kolodny EH, Logigian EL, Ullman MD. Nervous system involvement in Fabry's disease: clinicopathological and biochemical correlation. Ann Neurol. (1988) 23:505-9. doi: 10.1002/ana.410230513

37. Okeda R, Nisihara M. An autopsy case of Fabry disease with neuropathological investigation of the pathogenesis of associated dementia. Neuropathology (2008) 28:532-40. doi: 10.1111/j.1440-1789.2008. 00883.x

38. Cacciari E, Milani S, Balsamo A, Spada E, Bona G, Cavallo L, et al. Italian cross-sectional growth charts for height, weight and BMI (2 to $20 \mathrm{yr}$ ). J Endocrinol Investig. (2006) 29:581-93. doi: 10.1007/BF033 44156

Conflict of Interest Statement: SC received fees for speaking from Genzyme. AB received travel grants for participation in scientific meetings on contrast media by Bracco and user meetings by General Electric. VBM reports personal fees from Novartis, Biogen, Genzyme, Teva, Almirall, Bayer, and Merck. ER received travel grant and honoraria for serving as consultant to Genzyme and Shire. ET received fees for speaking from Scientific Press, Ars Educandi, and Shire Italy. AP received travel grant and honoraria for serving as consultant to Genzyme, Shire and Amicus.

The remaining authors declare that the research was conducted in the absence of any commercial or financial relationships that could be construed as a potential conflict of interest.

Copyright (C) 2018 Pontillo, Cocozza, Brunetti, Brescia Morra, Riccio, Russo, Saccà, Tedeschi, Pisani and Quarantelli. This is an open-access article distributed under the terms of the Creative Commons Attribution License (CC BY). The use, distribution or reproduction in other forums is permitted, provided the original author(s) and the copyright owner(s) are credited and that the original publication in this journal is cited, in accordance with accepted academic practice. No use, distribution or reproduction is permitted which does not comply with these terms. 